\title{
MODULAR DESIGN FOR SPACE ENGINEERING RESEARCH PLATFORMS
}

\author{
Mark Post, Regina Lee, Brendan Quine \\ Earth and Space Science and Engineering, York University, 4700 Keele St., Toronto, Canada \\ markpost@yorku.ca,reginal@yorku.ca,bquine@yorku.ca
}

Keywords: field robotics, mobile robots, embedded systems, electronics

\begin{abstract}
In this paper, we describe a modular design approach that is used in developing several research platforms for space engineering research at York University. We briefly describe three distinct research projects for space science and engineering research: a 6kg micro-rover under development for the Northern Light mars lander mission, a 50kg rover built by the York University Rover Team to compete in the annual University Rover Challenge, and a 1U CubeSat design for nanosatellite technology development research. All three research projects share a design philosophy to achieve modularity, efficiency, robustness and simplicity by adopting a common embedded hardware configuration using COTS hardware and a simple control topology. An on-board computer board stack based on a PC/104 or similar form-factor provides centralized control using an ARM microcontroller. Payloads and application-specific components are added using robust SPI, synchronous serial, and RS-485 interfaces, with provision made for ethernet and USB connectivity when needed. The software for this system is is based on open-source compilers and operating systems and is also modular in nature, using a portable base station GUI, wireless mesh networking between different systems, and JAUS messaging for flexible component-level communications. The proposed design approach allows sharing of resources while optimizing design features with cost-effective, readily-available commercial components for complex research projects.
\end{abstract}

\section{INTRODUCTION}

The challenges of creating system designs for space engineering research and education are often different from those encountered in industry. To minimize costs and development time, commercial-offthe-shelf (COTS) components and free open-source software (FOSS) tools are preferred for building systems for use in research and student projects. Additionally, the engineers tasked with building and programming the robotic hardware are often students with limited knowledge of embedded systems and minimal hands-on experience, so using simple interfaces and design methodologies is generally beneficial. In many cases, it is necessary to integrate networks of simple components in a manner that not only keeps communications simple and efficient for prototype robots such as in (Candini et al., 2009), but also to allow sufficient flexibility for enlarging and upgrading the systems later.

This approach must be balanced by the necessity to design and build reliable systems that can tolerate potentially hostile environments while operating. Noise tolerance, thermal limitations, and ESD protection need to be considered for the system to be robust in practical use. As many universities, including York University in Toronto, are now participating in the development of microsatellites and nanosatellites, such systems may also need to be operable in the extremes of vacuum, temperature and radiation in outer space. Successful nanosatellite missions using off-the-shelf hardware such as CanX-2 (Sarda and C., 2010) have proven that given good engineering practice, it is not always necessary to use very specialized hardware to operate in hostile or distant environments, and depending on the mission, simple radiation shielding and temperature control may be sufficient. 


\section{DESIGN CRITERIA}

The following four criteria are proposed for the development of an adaptable, mobile robotic research platform.

1. Modularity: Parts of the system can be added and removed as needed for the purpose at hand, but the system uses common modules and interfaces for multiple purposes.

2. Efficiency: Most space hardware is solarpowered, and most mobile robotic hardware is battery-powered, so minimizing power use and weight is essential.

3. Robustness: Components and bus systems have to tolerate environmental variations and extremes that arise from the variety of conditions that may be encountered.

4. Simplicity: For a research institution, hardware must be simple to understand and flexible in operation so that it can be applied to many different levels of projects.

Recently, the rapid development of low-cost microcontrollers and highly-integrated logic devices for the mobile device market has made it possible to build modular, general purpose robotic hardware for a fraction of the cost and complexity needed even two decades ago. Designing and constructing these systems in-house has the benefits of easy modification, low cost, and a better overall understanding of the system's dynamics, and students can work in depth with the system as part of their education. To leverage this capability, we propose a modular electronic design philosophy for electronic systems that can be used in a variety of research projects. The hardware should be easily adaptable to different uses. System modules should be easily field-replaceable and serviceable. Electronic parts used in this system need to be readily available for ease of development, tolerant of noise and voltage in field conditions, and inexpensive. Components in ball-grid array and other no-lead packages should be avoided to facilitate soldering, repair, and improve vibration resistance. Programming should make use of open and freely-available tools to ensure continuing availability.

\subsection{Current Implementations}

The motivation for designing a standardized set of interfaces and implementation guidelines is that differ- ent research groups with varying hardware requirements can share designs, hardware, and program code so more time and effort can be spend on the research itself as opposed to the hardware necessary to accomplish it. This paper describes a framework for field robotics that lends itself well to modular robotic systems such as rovers with payloads, unmanned aerial vehicles, sensor networks, and even nanosatellites. The focus is on efficiency and minimization so that physically small and solar-powered systems can be built, but the embedded hardware is centered on a modern ARM microcontroller that is powerful enough to support a variety of autonomous operating modes. This modular design is being put to use in several student and research projects at York University.

Foremostly, a micro-rover (or " $\mu$ rover") prototype known as the Beaver is currently under development for use in the Northern Light Mars Lander mission (Quine et al., 2008), a Canadian Mars lander and rover system instrumented to provide new information on the Martian atmosphere, surface, and subsurface. The $6 \mathrm{~kg}$ micro-rover is capable of autonomous operation and is built on a four-wheel drive swing-arm suspension chassis with two science payload spaces at the front and back. The first version of the Beaver was tested at the Algonquin Radio Observatory in November 2010, and the results of testing have confirmed the usability of the modular system. The uRover design is based on a $10 \times 10 \times 20 \mathrm{~cm}$ form factor to accommodate PC/104 form factor boards, batteries, and associated hardware, and the on-board computer is based on a low-power ARM microcontroller that uses routed messaging for inter-component communications. A research prototype is shown in Figure 1 (a).

Also, The York University Rover Team (YURT) is a student group that has achieved podium finishes for all three years competing in the University Rover Challenge held by the Mars Society. Each year, the team builds a new $50 \mathrm{~kg}$ rover based on the experience gained in previous years and uses it to perform four distinct remotely-guided tasks in desert conditions with rocky terrain and $>30^{\circ} \mathrm{C}$ heat at the Mars Desert Research Station in Utah, USA (Post and Lee, 2011). The modular system design described here has both influenced and been influenced by the experiences of YURT, and each generation of rover refines the standards and implementation of the previous one. Figure 1 (b) shows the rover developed for the 2011 competition, known as E.V.E. (Enhanced Vehicle Explorer)

Finally, the nanosatellite development program at York University is in the process of designing a $1 \mathrm{U}$ CubeSat (of size $10 \times 10 \times 10 \mathrm{~cm}$ ) that follows the 
modular embedded design philosophy. For a typical CubeSat application, external payload interfaces and command routing is not necessary, as every component is contained on the on-board computer stack. The components are different but the engineering can remain consistent and retain most of the compatibility with other robotic systems. A conceptual view of the CubeSat when completed is shown in Figure 1 (c). Planned future work includes development of unmanned aerial vehicles similar to those described in (Cho et al., 2007) using the same modular system.

\section{HARDWARE ARCHITECTURE}

To achieve the described flexibility in configuration of the the embedded on-board computer (OBC) hardware, a stack of printed circuit boards (PCB) is proposed as the basis for the modular system. This OBC stack includes a common motherboard for centralized control, and additional daughterboards that can be added to suit the application. If more complexity is needed, additional stacks can be added and linked together.

A diagram of proposed component and interface organization in the modular system is shown in Figure 2. To date, most implementations have been built by hand using through-hole components, which makes the motherboards, daughterboards and payloads larger and heavier. A surface-mount electronic implementation in PC/104 form factor is currently undergoing critical design review, and will be used for the micro-rover prototype, while a slightly different but compatible implementation is under development for the nanosatellite prototype. The PC/104 form factor describes a stack of PCBs with pass-through $0.1^{\text {“ }}$ 64-pin and 40-pin headers, and was chosen because it is a common standard for embedded systems in industry and robotics, and strikes a good balance between compactness and flexibility. The pinout is altered to contain the communications interfaces described here. The resulting board stack will look as shown in Figure 3.

\subsection{Control Topology}

The proposed modular system is built around a powerful central microcontroller on a single motherboard in the OBC stack that is responsible for autonomy and system coordination. Additional daughterboards can contain a set of small component microcontrollers that perform low-level tasks and can be customized to suit different requirements. To make most effective use of off-the-shelf components, the interfaces used

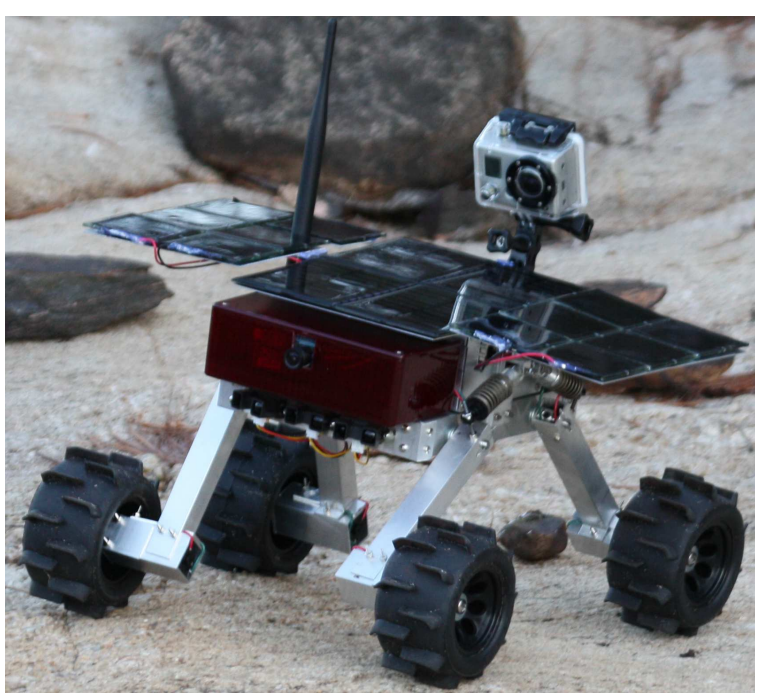

(a) Beaver micro-rover prototype

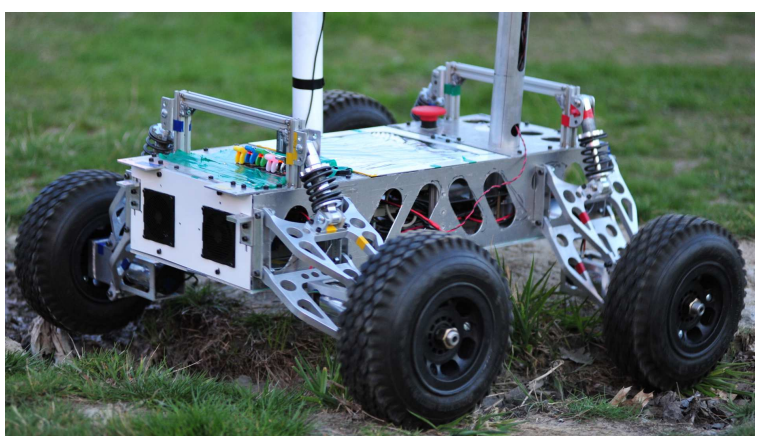

(b) YURT 2011 competition rover E.V.E.

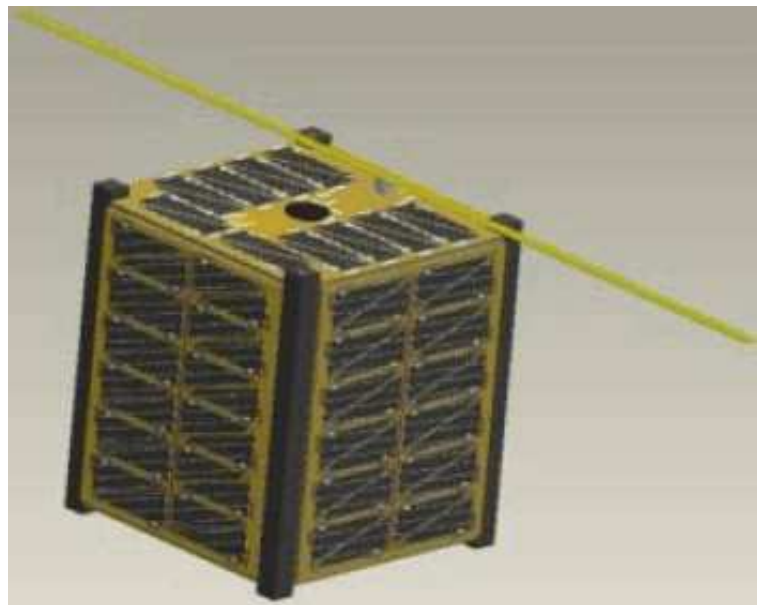

(c) York CubeSat nanosatellite concept

Figure 1: Existing implementations of modular system 


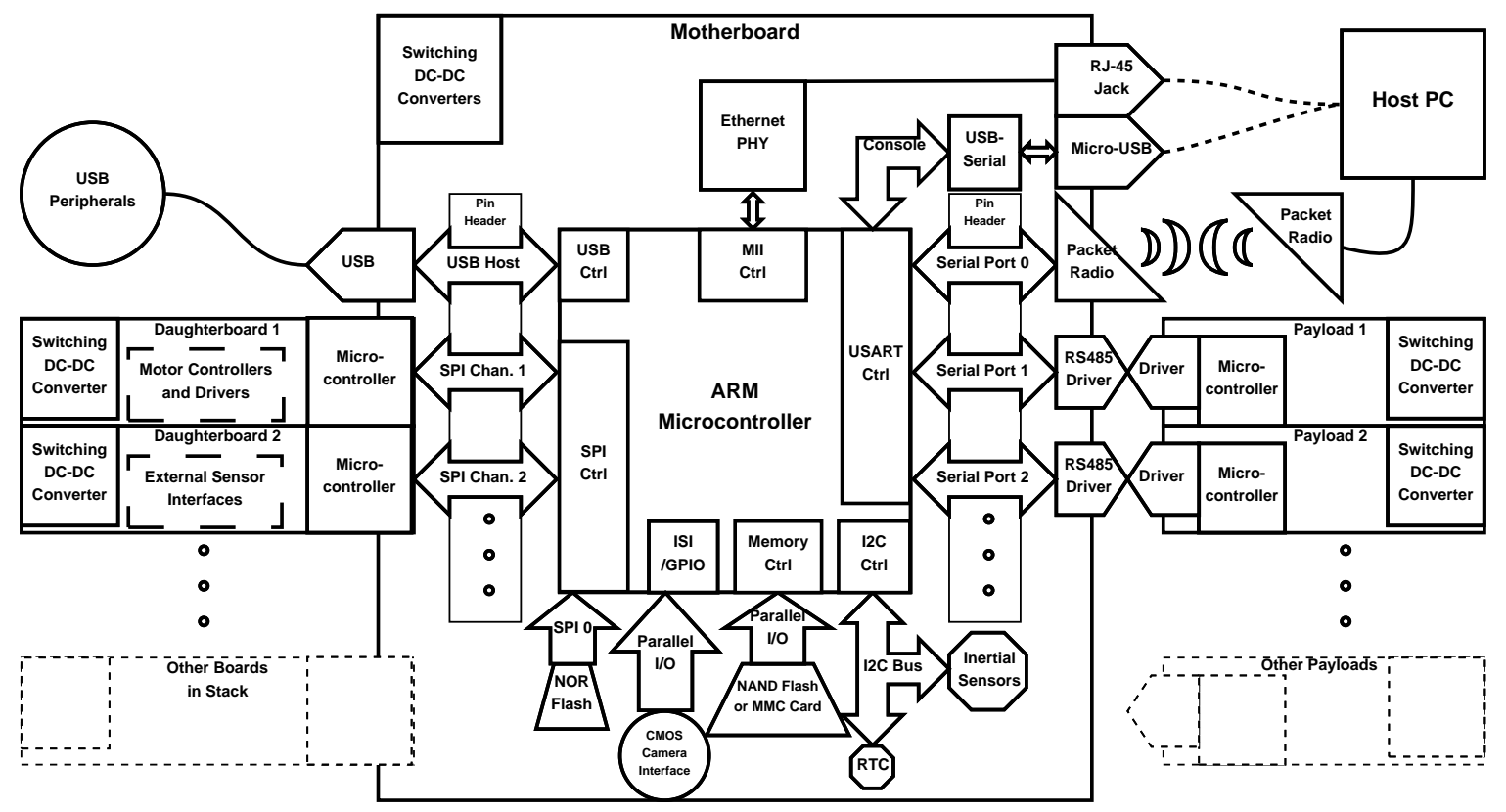

Figure 2: Diagram of modular system topology

by rover systems have to be common enough to be present on most modern embedded hardware, but still well-suited for real-time robotic applications.

The standardized ARM architecture is preferred for central microcontrollers as it is possible to use embedded operating systems and program source code that are easily ported between specific microcontrollers, with only the low-level hardware interfaces requiring modification in some cases. In the case of embedded Linux, these low-level interfaces are generally handled in the kernel and accessible by means of user-space device interfaces. Currently, the Atmel AT91RM9200 and AT91SAM9260 ARM9 microcontrollers are used, though a variety of automationoriented ARM microcontrollers are available, most notably the Cortex-M3 and M4 series that use the Cortex Microcontroller Software Interface Standard (CMSIS), and will be used in future implementations. Due to the master-slave paradigm used in the modular system, only one central ARM motherboard is usable in each OBC stack, but this limitation has not caused problems as ARM microcontrollers comparable in power to desktop computers are now available, and multiple OBC stacks can be used on a vehicle if needed. As per CubeSat standards, the 1U CubeSat under development at York University is based on the PC/104 form factor with an ARM-based custom OBC stack. The YURT rovers use an ALIX embedded router motherboard with external serial interfaces rather than an ARM microcontroller and hard- ware stack, but future implementations may use ARM systems for better power efficiency and higher integration with other systems.

The component microcontrollers can be inexpensive 8-bit microcontrollers for motor control, sensor monitoring, and payload management, or more complex controllers if required. The microcontroller most often chosen for this role is the Atmel AVR 8-bit RISC architecture, which is easily in-system programmable using the $\mathrm{GNU} \mathrm{C} / \mathrm{C}++$ compilers and open-hardware SPI programmers. Modularity is achieved by attaching these to the central ARM microcontroller using simple serial and parallel bus standards and pin headers, or D-sub connectors for external connections. The use of small microcontrollers in this manner allows hardware customization without having to change the central controller in the system or its motherboard, which is often the most complex and costly component in a small robotic system.

To maximize reliability, "multiple-hop" communications are avoided. SPI channels are connected directly to the central microcontroller and serial interfaces are connected via a MAX489 or similar serial buffer IC with high voltage and ESD protection. Damage to robotic components, which is often caused by ESD and electrical shorts, is isolated by means of the communication buffers and is less likely to spread to adjacent components. Programmable logic devices such as FPGAs and CPLDs is avoided, since these devices often require proprietary software and 
hardware to program, and can consume significantly more power than microcontrollers of similar capability. However, low-power CPLD devices can be used where microcontrollers are less well-suited, such as high-speed encoding and decoding tasks.

\subsection{Board-to-Board Communications}

All signals are carried between OBC boards as shown in Figure 3 via standard 0.1 " pin headers. This facilitates connection between boards and wires, and makes debugging easy. Proprietary connectors are often hard to test and usually are not compatible between manufacturers and sizes. Due to the resiliency of using separate transmit, receive, clock, and frame synchronization wires, SPI is preferred for board-toboard communication at high bit rates, though a synchronous serial interface with clock like also provides a reliable connection. Communication with payloads is generally achieved using 8-bit serial communications, which is the most common low-level way to interface with embedded systems, but TTL serial interfaces are also broken out through the pin headers for use in used in board-to-board communications. For debugging and system recovery purposes, one serial port should be designated as a system console or debug port, and this is usually available via USBto-Serial converter IC for convenience in connecting portable PCs. GPS Devices and embedded radios are often serial devices that can be included in the OBC stack. Though very common, high speed personal computer buses such as PCI and PCI-Express do not present significant benefit to low-speed embedded systems with limited I/O resources and integrated controllers.

The number of Serial and SPI devices is only limited by the number of SPI and Serial interfaces on the ARM microcontroller. Provision is made on the board headers for at least four serial interfaces and four SPI interfaces with chip selects. Board headers do not make use of line drivers to save power. There have been no problems using direct pin connections between boards so long as the housing for the OBC stack is sealed from sources of ESD and contaminants. The York CubeSat manages all onboard systems through a mixture of SPI and synchronous TTL serial interfaces.

For register-level access to onboard sensors and peripherals, $\mathrm{I}^{2} \mathrm{C}$ is a synchronous bus widely used for register-level communications to multiple embedded ICs, but because it must embed addressing, bus arbitration, and multi-master capability using only two wires and a simple protocol, it is very sensitive to interference and noise from the environment. To maxi-

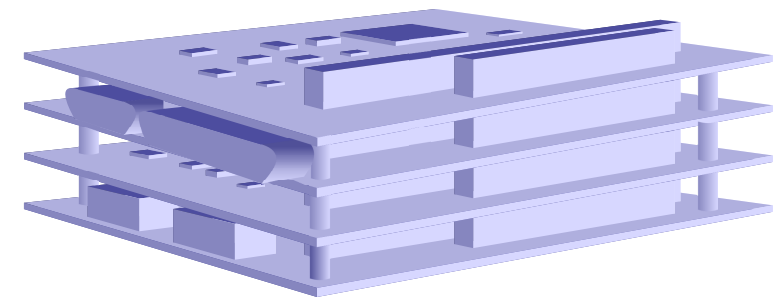

Figure 3: Rendering of PC/104 stack as used on micro-rover

mize reliability, trace lengths to the $\mathrm{I}^{2} \mathrm{C}$ host must be minimized and daughterboards and payloads must use a microcontroller to transfer data to SPI or serial bus lines from the $\mathrm{I}^{2} \mathrm{C}$ device.

For interfacing high-bandwidth devices, CMOS and CCD cameras are interfaced directly to the central ARM microcontroller via either a parallel bus of general-purpose input-output (GPIO) pins or if available, a dedicated image sensor interface (ISI). Both volatile (RAM) and non-volatile (Flash) memory is interfaced by means of dedicated memory hardware, i.e. RAM interface, NAND Flash interface, and SDIO bus. Nearly all modern ARM microcontrollers have dedicated memory interfaces.

\subsection{Payload Communications}

For external payload communications, RS-485 has been selected as the standard of choice. The RS-485 interface is an industry standard for differential-pair serial communications to multiple transceivers, and is already used on many robotic systems. It may be used in either half-duplex mode (separate transmit and receive pairs) or full-duplex mode (pairs connected), but full-duplex with a single master is preferred to avoid byte collisions and the need for bus arbitration. RS-422 devices are also compatible with this interface.

Additionally, the RS-485 interface is designed for bidirectional communication with up to 32 devices on a single bus, the number of potential payloads is high enough to accommodate large robotic systems. The 2011 YURT rover uses an RS-485 bus to control six arm motor control boards from a single serial interface. While RS-485 can operate at 10 megabits per second at 40 feet and 100 kilobits per second at 4000 feet, to allow low-speed microcontrollers to communicate reliably, the standard baud rate of 115200 baud is used as the default speed unless higher bandwidth for the specific payload is required. For long-distance disconnectable operation, an RJ-45 jack and twisted-pair cabling also provides good performance. For extreme environments, an additional 
pair is used to transmit a clock signal for synchronous operation to ensure reliable transmission. With the exception of high-bandwidth devices such as optical sensors, memory, and media interfaces, these serial links are more than capable of real-time command transmission. As no standardized D-sub pinout has been accepted for RS-485 communications, one was created to suit the modular system with differential clock (CK) power supply, and interrupt pins. The external interfaces are situated on a board in the OBC stack with line drivers and external DE9 connectors. To pass GPIO signals and parallel buses, DB25 connectors are useful with each pin buffered against ESD and high voltage also.

The very common RS-232 serial port, while still present in a huge number of devices, is not traditionally compatible with RS-485 interfaces and is less efficient as it is based on negative logic and $\pm 5-15 \mathrm{~V}$ signal levels. A dedicated converter is the best method for interconnection. However, given that most modern hardware uses only $\pm 5-7 \mathrm{~V}$ and has thresholds at $1 V$, it is possible for RS-232 and RS-485 devices to communicate directly if required, for example, in new hardware testing or field debugging using RS-232. The payload pinout, shown in Figure 4, was chosen to be electrically compatible with RS-232 for this reason and to increase safety in case of accidental RS-232 connection. The RS-485 TX-(Z) and RX-(B) pins are connected to RS-232 RX and TX respectively to invert logic levels, $\mathrm{TX}+(\mathrm{Y})$ and $\mathrm{RX}+(\mathrm{A})$ are connected to RS-232 CTS and RTS respectively to serve as a ground reference. The CTS and RTS pins must be allowed to float to ground, and if the hardware does not allow this, $\mathrm{TX}+(\mathrm{Y})$ and $\mathrm{RX}+(\mathrm{A})$ must be connected to the RS-232 ground instead. This method works well using a MAX489 transceiver IC and is considered an option for debugging and testing with RS-232 hardware and purpose-built serial interfaces. But it is less efficient than only RS-485 communications and breaks the RS-232 specification by referencing signal levels to ground rather than $\pm 5-15 \mathrm{~V}$. Newer RS-232 hardware and USB to RS-232 converters that have low signal voltages are useable, but older highvoltage RS-232 hardware may be unsafe to use as the RS-485 transceiver used must be able to tolerate the signal voltages.

For devices other than system payloads, USB can be used for COTS hardware, since the interface is present on many embedded devices, but is generally restricted to hot-pluggable external hardware and mass storage devices rather than hard-wired onboard peripherals. Ethernet can also be used, but has significant programming and routing overhead, so it is preferred as an external network interface for de-

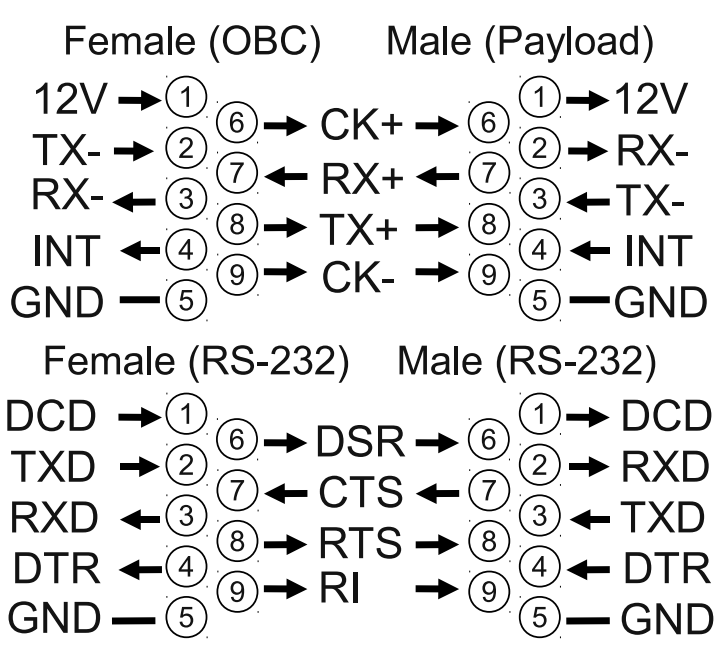

Figure 4: Payload DE9 connector and RS-232 pinouts

velopment purposes. Other industrial standards for communications such as CAN, LIN, Firewire and Spacewire, though often used in other systems for reliability-critical applications (Torre et al., 2010), are not present on the majority of embedded devices and are not generally used in the interest of easy interoperability. The Spacewire bus in particular, designed and used for space hardware, is very fast and robust, but is only implemented in hardware on a very small set of radiation-hardened devices which are very rare and expensive.

\subsection{Radio Communications}

Wireless communications between mobile robots and control stations is a critical component in applications requiring remote control or monitoring. To simplify terrestrial work, it is desirable to use license-free ISM bands or amateur radio bands when communicating. The frequency must also be high enough to support high bit rate communications, preferably at least the basic system rate of 115200 baud. The $2.4 \mathrm{GHz}$ band is by far the most popular, but higher frequency systems have lower range and worse non-line-of-sight (NLOS) characteristics, and this band has also become very crowded with interference from consumer electronic devices. Another option is the $433 \mathrm{MHz}$ band and below, which can be used by amateur radio operators, but limits data rates on most radio hardware to 38400 baud and lower. The $900 \mathrm{MHz}$ ISM band is a good medium between these options, as it provides reasonable range and NLOS characteristics, can support bit rates above 115200 baud, and is not used excessively by consumer devices. 
Another consideration is the need for multi-point communications and routing. There are many situations when several mobile robots need to stay in communications with each other and with base station units, but relative movement, terrain variations and ambient conditions make static network structures unreliable. One popular solution to this is the mesh network, which allows multiple transceivers to route data to each other using dynamically maintained and self healing network structures. The best known industrial mesh network specification is ZigBee, which is used frequently in sensor mesh networks but requires a dedicated coordinator and router nodes which limits the flexibility of the network. The B.A.T.M.A.N. (better approach to mobile ad-hoc networking) routing protocol was recently added to the Linux kernel to allow mesh networking over wireless LAN networks, but is not designed for low-level serial hardware.

To provide a long-range serial mesh networking system, the Digi XBee PRO Digimesh 900 serial radio module is used frequently on modular research hardware, one exception being the YURT rover which requires high camera data rates and uses Ubiquiti XR9 900MHz mini-PCI network cards, with XBee modules as a fallback if needed. The Digimesh protocol is proprietary, but allows mesh networking without a central coordinator. As these radio modules use a well-known form factor, replacing them with $2.4 \mathrm{GHz}$ modules or custom modules that change frequency, range, and protocol is simple.

Future work in this area includes the development of an open-hardware transceiver module and purposedesigned mesh network protocol. For higher-power radio systems, an OBC daughterboard can be used. The advantage of using simple serial communications rather than Ethernet, Wireless LAN, or other IP communications standards, is that a wider variety of radio systems and communications frequencies can be used, up to and including S-band and UHF radio systems for space hardware. It is also easy to encapsulate serial communications over IP-based systems if desired.

\subsection{Power}

Onboard batteries are expected to be used for power storage, and can be charged from solar panels or other sources. For small rovers and aerial vehicles, a single cell or stack of lithium-ion (Li-Ion) cells in series must be used to achieve high power densities. Since the batteries must be balance-charged for longevity, the electronics can run off the bottom cell in the stack, which is done on the micro-rover prototype. For large rovers, nickel metal hydride (NiMH) battery packs can be used for higher stability and environmental tolerance, and $24 \mathrm{~V}$ is used for powering larger drive motors with the rest of the electronics either being powered by a separate $12 \mathrm{~V}$ battery as previous YURT rovers have been, or by using heavily-filtered switching converters tolerant of high voltages. Battery voltage and current monitoring is done via analog-todigital converters, which are present on most modern microcontrollers and ideally are present on the central ARM microcontroller. In-depth testing of Li-Ion cells (Navarathinam et al., 2011), NiMH battery packs and high-current DC-DC power converters has been done to verify performance in extreme environmental and operational conditions.

With the availability of low-cost highly-integrated switch-mode converters, it is feasible for each module in the system to convert DC power from the system battery to whatever level is needed. Daughterboards in the OBC generally use the system battery for power as well as regulated $3.3 \mathrm{~V}$ and $5 \mathrm{~V}$ supply rails provided by the motherboard. The choice of power supply is important. Simple linear voltage regulators that are commonly used on microcontroller boards become less efficient relative to the input/output voltage difference, and are unsuitable for use in vacuum or high-temperature environments as the only mechanism for cooling is direct radiation of heat. To achieve the highest power conversion efficiency possible, switching step-down (buck) converters are used, if at all possible, rather than stepup (boost) converters. Using only low-dropout buck converters with high input voltage tolerance makes it possible to achieve system efficiencies close to $90 \%$. Also, each module is responsible for filtering its own load noise via capacitor-inductor networks to avoid injecting power supply transients back into the OBC. Zener diodes should also be used to protect from ESD and over-voltages.

\subsection{Motor Control}

An essential element of most robots is electronic motor and actuator control. Traditionally, brush DC motors with mechanical commutation have been used for robotic movement due to their cost-effectiveness and simplicity of implementation, and brush DC motors are still dominant in the marketplace. However, the recent availability of high-speed microcontrollers and integrated drive bridges have made brushless DC motors, which require external electronic commutation, increasingly popular. Brushless DC motors have generally higher efficiency and longevity due to the lack of mechanical brushes and are preferred for hazardous environments and space applications, but re- 
quire a different control method usually considered incompatible with brush DC (Lee et al., 2003).

Ideally, both BLDC and DC motors could be driven with only software changes, so any changes to the system could be made by a microcontroller. Many small microcontrollers are suitable for implementing feedback and control of motors, and some PIC and AVR models include dedicated hardware for this purpose, while still being programmable in $\mathrm{C}$ and using minimal power. Flexible designs for multiple motors have been achieved using FPGA hardware and DSP ICs, such as in (Zerigui et al., 2007). While this approach provides high control speeds, it requires more power to run the FPGA and introduces significant complexity in design and programming. To enable a common system to drive both brush DC and brushless DC motors, a hybrid approach is required. DC motors are usually controlled by using a fixed input and pulse-width modulation (PWM) to manage the amount of total power provided to the motor. Brushless DC motors are controlled by shunting power to different motor coils (or "phases") one at a time in synchronization with rotor speed, usually measured by Hall sensors, and speed control is achieved by changing the time that power is shunted. Both systems usually use field-effect transistors (FETs) in a half-bridge configuration to control current flow, two for a brush motor in the "H-bridge" configuration, and one for each phase of a brushless motor. Brushless motors usually have up to three phases, so a hybrid controller can be built with three such half-bridges.

Figure 5 shows the hybrid system in both brushless DC (a) and brush DC (b) configurations. For three-phase brushless use, a single set of three halfbridges are cycled in sequence to supply power to the three motor phases, while for brush use, two of these form an H-bridge to supply pulse-width modulated DC power to the motor. Control feedback is via a 3-phase Hall sensor in the brushless case and bridges are commutated by setting GPIO lines, while a quadrature (Gray code) encoder is used in the brush case and pulse-width modulation control is used in hardware. The drive controller $12 \mathrm{~V}$ is assumed to be the drive voltage in this diagram, but any appropriate motor voltage can be used. Each motor requires the use of one set of drive bridges, though a fast microcontroller can usually drive more than one bridge at a time. For small mobile robots and rovers, all needed drive motor controllers can usually be implemented on an OBC daughterboard, but for larger robots, the drive controller is implemented as an external payload to gain more space and better isolation. A prototype of this hybrid system has been built for the microrover, and shows promise for larger systems as well.

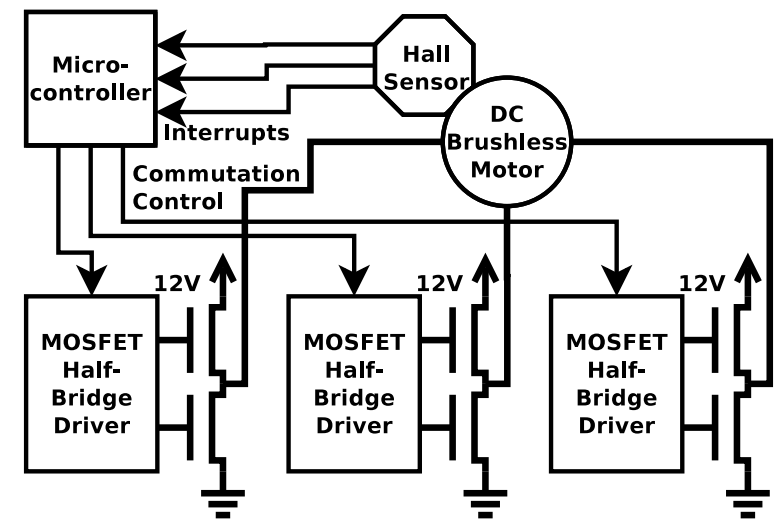

(a) Brushless Motor Configuration

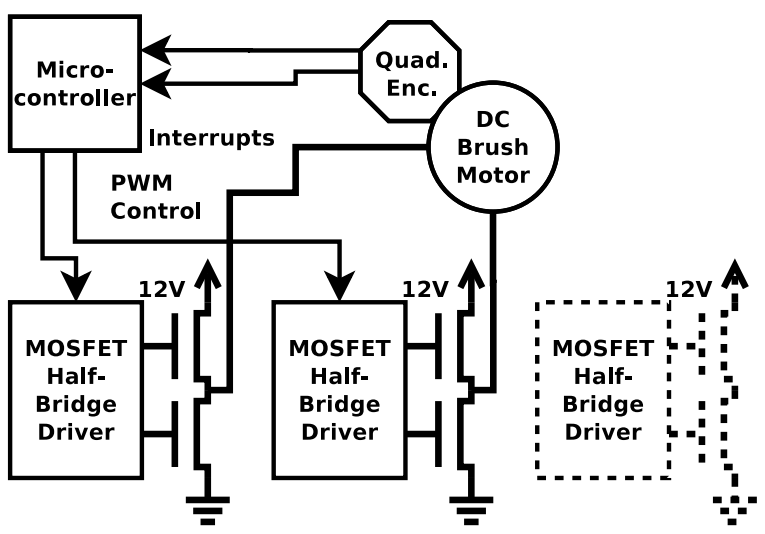

(b) Brush DC Motor Configuration

Figure 5: DC motor controller basic configurations

\section{SOFTWARE ARCHITECTURE}

In order to benefit from and support the opensource community, and to ensure that the basis for the system remains freely available and up-to-date, open-source OS software and the GNU compiler collection (GCC) is preferred to form the software base for the modular system. It is assumed that most mobile platform software will have to control actuators, read sensors, and communicate with a base station, a set of central control programs for autonomous operation, or both with system coordination occurring on the central ARM microcontroller. The YURT rover (Post and Lee, 2011) and micro-rover prototype are good examples of this kind of a system, as is the example is given in (Marosy et al., 2009). As such, a robust multitasking framework and a routing and command handling system are essential to reliable operation.

The software for the system is stored in NAND flash memory on the microcontrollers and on redundant NAND and NOR flash devices on the mother- 
board. NAND flash is the most common and provides large storage capacity, while NOR flash devices can store only a few megabytes but may be more resistant to radiation and corruption (Farokh Irom, 2008). To mitigate the risks of software corruption, in the event of a boot failure the bootloader for the central ARM microcontroller will switch between two kernel/filesystem images. It is possible for the central ARM microcontroller to boot from the smaller failover device and retain sufficient capacity to reprogram internal component microcontrollers as required using SPI. Implementation of error-correction codes is also proposed for improving storage reliability, though it would have to be done either in software or on an external logic device such as a programmable logic device (PLD). For NAND flash devices, the recent addition of UBIFS to the Linux kernel provides error detection and wear-leveling for NAND devices, and currently appears to be the best option for NAND devices in Linux.

\subsection{Operating Systems}

Direct-to-hardware programming on the central ARM microcontroller is possible, but an embedded operating system (OS) is usually used in research applications to to expedite software development and simplify hardware interfacing. The Emdebian Linux OS has been used for most of the existing development work, and other FOSS systems are being investigated for real-time flight hardware implementations such as Linux with Xenomai for real-time support and FreeRTOS as a standalone real-time framework.

Customized Linux kernel patches and drivers that implement user-space support for the embedded OBC peripherals such as the RS-485 interfaces, SPI controllers, and I2C sensors have been developed to provide hardware integration, and a set of dedicated libraries for 8-bit AVR microcontrollers has been created to assist in programming component applications for this system. The software for onboard systems has been developed entirely in $\mathrm{C}$ and $\mathrm{C}++$ for efficiency and minimal code size. Other languages can be run on resource-constrained hardware such as real-time Java, and using a Linux environment enables the use of Python as well. These will be retained as options for future research.

For overall control and monitoring of remote systems at the base station, a graphic user interface (GUI) has been developed using Java, with the philosophy that it must be portable to whatever OS is used at the base station, but need not run on resource-constrained embedded hardware. The GUI development is based on the work of the York University Rover Team, who have been steadily improving their base-station GUI concept for each generation of remotely-controlled rover. The GUI integrates system health monitoring, GPS localization, joystick movement control, and camera displays for convenient use of the system operator.

\subsection{JAUS Messaging}

For designs where only a few microcontrollers are fixed in connection such as most nanosatellites, simple byte-by-byte protocols work fine, but for large systems with multiple, changeable interfaces, a flexible message routing and communications protocol is necessary. The routing system and protocol used currently for robotics is based on the Joint Architecture for Unmanned Systems (JAUS), a messaging system that groups similar or associated capabilities of a system together and provides a consistent method of organizing subsystems (The joint architecture for unmanned systems working group, 2007). The JAUS structure can be considered close to the minimum of functionality for universal point-to-point communications in a multi-device system. Although JAUS does not specify implementation details, the JAUS reference implementation has been used as the basis for the OpenJAUS project, an open-source framework for remote operation of robotic systems that is portable to a wide range of platforms.

The JAUS architecture is a tree structure, where an entire system is composed of subsystems, each containing nodes, each containing components, which can have several redundant component instances. For the architecture we are describing, an individual robot represents a subsystem, a central ARM microcontroller represents a node and a microcontroller attached to that controller represents a component, which services a finite set of commands and may have redundant systems on the same bus. More than one central controller node can be present on a robot, and each node must run a node manager for routing communications to components, which is currently implemented as the OpenJAUS node manager. As JAUS commands can be large in scope and parsing requirements, microcontroller components can use custom small-footprint commands for simple tasks. By using only a small set of commands for each microcontroller, most components are capable of sending and receiving basic commands using JAUS natively, and additional functionality is easy to implement.

The advantages of JAUS messaging between components is that each node or component can initiate messages to any other part of the system. While control is usually centralized in autonomous robots, the 
capability of "bypassing" the central controller and communicating directly between components using only the node manager is sometimes useful and provides failover capabilities. However, the node manager on each node must be very stable and should be restarted if abnormal program behavior is noticed. For ease of debugging, a "console" mode is used on microcontrollers with sufficient flash memory, so that by entering an escape sequence (commonly the AT escape sequence "+++") a simple serial text console can be used to send commands and check the status of the microcontroller using ASCII keystrokes. This allows easy field testing of components without requiring a full JAUS implementation to be present. Development of JAUS-compatible code for the microrover prototype is proceeding, and may be used in the YURT rover in future as well.

\section{CONCLUSION}

In this paper, we briefly introduced three distinct space engineering research projects at York, namely micro-rover development, the York University Rover Team's larger scale Mars rover prototype design and a $1 \mathrm{U}$ CubeSat-based nanosatellite design. In all three distinct research projects, we aim to design mobile platforms with a level of autonomy and simplicity with distinct mission objects for each project. The micro-rover is designed for Mars environment with full autonomy and redundancy. The YURT rover is designed each year to complete at the University Rover Challenge with minimal autonomy, but with flexibility for four separate tasks. The $1 \mathrm{U}$ CubeSat is designed to demonstrate nanosatellite technologies currently under development at York University.

The common design approach we have taken in developing the above research platforms is the modularity and expandability in hardware and software design. We described a centralized control topology, in particular $\mathrm{OBC}$ design and communication protocols, and introduced a subsystem design methodology making use of highly-integrated commercial-off-theshelf (COTS) parts and low-cost components. In mobile robots, the motor controllers provide selectable brush or brushless DC motor management, while in a satellite platform, the same design approach can drive reaction wheels. A software architecture based on open components was also developed with modularity in mind. In mobile platforms, mesh networking and the JAUS structure was adopted to accommodate efficient point-to-point messaging communications. In future, we plan to implement the proposed modular design concept in other projects such as unmanned aerial vehicles and distributed sensors for navigation, mapping and remote sensing applications.

\section{REFERENCES}

Candini, G. P., Paolini, E., and Piergentili, F. (2009). Design and manufacture of a low cost educational hexapod rover. Acta Astronautica, 65(3-4):525 - 536.

Cho, A., Kim, J., Lee, S., Choi, S., Lee, B., Kim, B., Park, N., Kim, D., and Kee, C. (2007). Fully automatic taxiing, takeoff and landing of a uav using a singleantenna gps receiver only. In Control, Automation and Systems, 2007. ICCAS '07. International Conference, pages $821-825$.

Farokh Irom, D. N. N. (2008). Radiation tests of highly scaled high density commercial nonvolatile flash memories. Technical report, Jet Propulsion Laboratory, California Institute of Technology, Pasadena, CA.

Lee, J., Choi, J., Chung, J., and Lim, J. (2003). Design and implementation of integrated drive circuit for a small bldc motor. In Electrical Machines and Systems, 2003. ICEMS 2003. Sixth International Conference, volume 2 , pages $491-494$ vol. 2 .

Marosy, G., Kovacs, Z., and Horvath, G. (2009). Effective mars rover platform design with hardware / software co-design. In Design and Diagnostics of Electronic Circuits Systems, 2009. DDECS '09, pages 148-151.

Navarathinam, N., Lee, R., and Chesser, H. (2011). Characterization of lithium-polymer batteries for cubesat applications. Acta Astronautica, 68(11-12):1752 - 1760.

Post, M. and Lee, R. (2011). Lessons learned from the york university rover team (yurt) at the university rover challenge 2008-2009. Acta Astronautica, 68(78): $1343-1352$.

Quine, B., Lee, R., and Roberts, C. (2008). Northern light - a canadian mars lander development plan. In Conference Proceedings of CASI ASTRO 2008, Montreal, Canada.

Sarda, K. and C., G. (2010). Canadian advanced nanospace experiment 2 on-orbit operations; two years of pushing the nanosatellite performance envelope. In Conference Proceedings of CASI ASTRO 2010, Toronto, Canada.

The joint architecture for unmanned systems working group (2007). JAUS Reference Architecture Specification, Volume II, Part I. Version 3.3 edition.

Torre, A. D., Finzi, A. E., Genta, G., Curti, F., Schirone, L., Capuano, G., Sacchetti, A., Vukman, I., Mailland, F., Monchieri, E., Guidi, A., Trucco, R., Musso, I., and Lombardi, C. (2010). Amalia mission lunar rover-the conceptual design of the team italia rover, candidate for the google lunar x prize challenge. Acta Astronautica, 67(7-8):961 - 978 .

Zerigui, A., Wu, X., quan Deng, Z., and Yu, K. (2007). Common hardware design for $(2 n+2,0 * n * 3)$ wheels mobile rover. In Robotics and Biomimetics, 2007. ROBIO 2007, pages $712-716$. 